\title{
Photoluminescence and bowing parameters of InAsSb/InAs multiple quantum wells grown by molecular beam epitaxy
}

\author{
Po-Wei Liu, G. Tsai, and H. H. Lin ${ }^{\text {a) }}$ \\ Department of Electrical Engineering, National Taiwan University, Taipei 10617, Taiwan and Institute of \\ Electronics Engineering, National Taiwan University, Taipei 10617, Taiwan
}

\author{
A. Krier, ${ }^{\text {b) }}$ Q. D. Zhuang, and M. Stone \\ Department of Physics, Lancaster University, Lancaster LA1 4YB, United Kingdom
}

(Received 24 June 2006; accepted 9 October 2006; published online 17 November 2006)

\begin{abstract}
Detailed studies are reported on the photoluminescence of InAsSb/InAs multiple quantum wells grown by molecular beam epitaxy on InAs substrates with the Sb mole fraction ranging from 0.06 to 0.13 . From $4 \mathrm{~K}$ photoluminescence the band alignment was determined to be staggered type II. By comparing the emission peak energies with a transition energy calculation it was found that both the conduction and valence bands of InAsSb alloy exhibit some bowing. The bowing parameters were determined to be in the ratio of 4:6. For a sample with $\mathrm{Sb}$ composition $\sim 0.12$ in the quantum well the photoluminescence emission band covers the $\mathrm{CO}_{2}$ absorption peak making it suitable for use in sources for $\mathrm{CO}_{2}$ detection. (C) 2006 American Institute of Physics. [DOI: 10.1063/1.2388879]
\end{abstract}

Among the family of bulk III-V compound semiconductors the InAsSb alloy system has the lowest band gap energy $\left(E_{g}=0.145 \mathrm{eV}\right.$ at $\left.x=0.634\right)$ and is thus an important material for mid-IR devices. ${ }^{1,2}$ Heterostructures based on InAsSb alloys, such as InAs/InAsSb, InAsSb/InAsPSb, InAsSb/InGaSb, and so on, have been widely applied as the active medium for mid-IR optoelectronic devices. ${ }^{3-6}$ The InAsSb/InAs multiple quantum well (MQW) system is appropriate for use in the active region of 3-5 $\mu \mathrm{m}$ lightemitting diodes that could be deployed in portable gas analyzers and which are important for environmental protection. $^{6}$ In this letter we report on a series of InAsSb/InAs multiple quantum well samples grown by molecular beam epitaxy (MBE) on InAs substrates. Photoluminescence (PL) spectroscopy measurements were undertaken to investigate the dependence of emission wavelength on composition and also to gain information about the conduction band and valence band bowing parameters. The transition energies of the above-mentioned samples lie within the range of $0.2-0.4 \mathrm{eV}(3-5 \mu \mathrm{m})$.

The InAsSb/InAs MQW samples were grown on (100) $n^{+}$-InAs substrates using a VG-V80H MBE system. Two cracker cells were used to provide the $\mathrm{Sb}$ and $\mathrm{As}_{2}$ beams. A thermal effusion $\mathrm{K}$ cell was used to provide the In flux. An InAs buffer layer with a thickness of $100 \mathrm{~nm}$ was grown at $480{ }^{\circ} \mathrm{C}$. Then a seven-period InAsSb/InAs MQW with various $\mathrm{Sb}$ compositions was grown at $450{ }^{\circ} \mathrm{C}$. The thickness of the InAsSb well and InAs barrier were 7 and $50 \mathrm{~nm}$, respectively. In situ reflection high energy electron diffraction (RHEED) was used to monitor surface reconstruction. After growth the samples were characterized using double crystal X-ray diffraction (DXRD) and PL to study their structural and optical properties.

Figure 1 shows the DXRD spectra of the InAsSb/InAs MQW samples. During the growth of the MQWs, the in situ RHEED pattern showed a clear $4 \times 2$ reconstruction when InAs was deposited and became slightly blurry when InAsSb was deposited. This implies a rougher growing surface when

\footnotetext{
${ }^{a)}$ Electronic mail: hhlin@ntu.edu.tw
}

the $\mathrm{Sb}$ beam was incident. For samples with a higher $\mathrm{Sb}$ composition, only bulk lines were observed and we believe that this may result from the stress caused by lattice mismatch. However, the satellite peaks in the DXRD spectra of these samples show only $\sim 80$ arc sec linewidths, indicating a good crystal quality. The Sb composition of the MQWs in these samples was also determined from the DXRD measurements using simulations in the usual manner.

Figure 2 shows the low temperature (4 K) PL spectra of the various MQW samples. As the $\mathrm{Sb}$ composition increases, the peak wavelength exhibits a redshift in the range from 3.4 to $4.5 \mu \mathrm{m}$ and the peak intensity decreases. From the DXRD measurement, the highest Sb mole fraction in this series is 0.137 , for which a critical thickness calculation based on the Matthews-Blakeslee model $^{7}$ indicates a value of $57 \mathrm{~nm}$. This thickness is much larger than the total InAsSb thickness of $35 \mathrm{~nm}$ in our samples. Therefore, misfit dislocations should not be responsible for the intensity decrement in the samples with higher Sb composition. The good crystallinity shown in Fig. 1 also supports this point. It is known that strained InAsSb/InAs MQW on InAs substrate exhibits

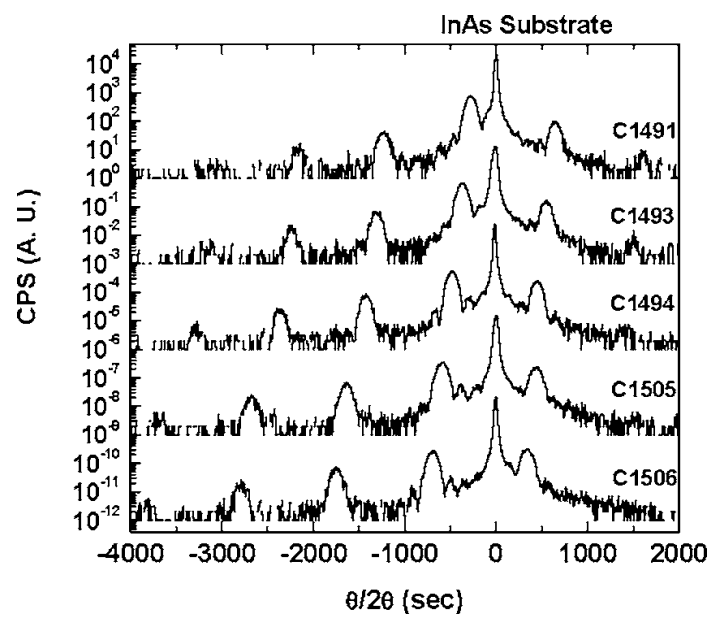

FIG. 1. DXRD spectra of InAsSb/InAs MQWs with different $\mathrm{Sb}$ compositions. 


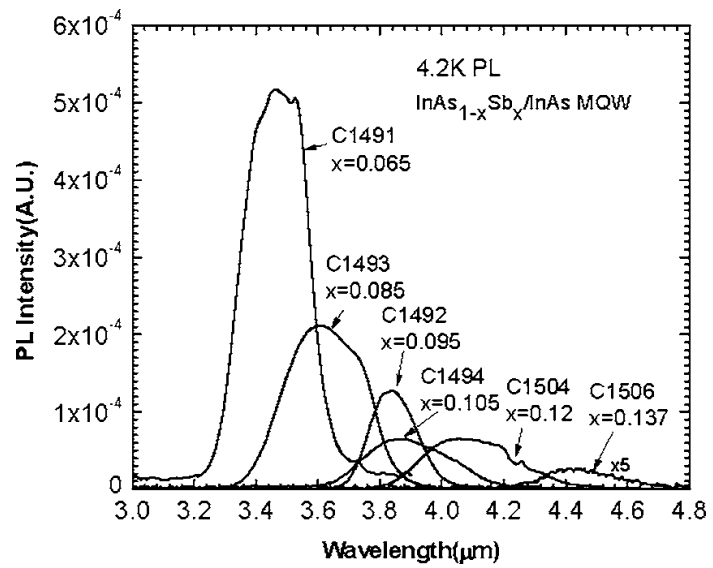

FIG. 2. $4 \mathrm{~K} \mathrm{PL}$ spectra of InAsSb/InAs MQWs with different $\mathrm{Sb}$ compositions.

a staggered type-II band lineup. ${ }^{8}$ The conduction band minimum of InAsSb is higher than that of InAs, and the electrons will reside mostly in the InAs barriers. But, because the conduction band offset is small the electron wave function spreads out. Since the holes are more strongly confined in InAsSb, the samples with higher $\mathrm{Sb}$ mole fraction will have lower wave function overlap, a lower dipole matrix element, and therefore a lower recombination rate. A simple calculation solving for the electron and hole wave functions of the flatband structure was made and showed that the intensity ratios between the samples are in reasonably good agreement. For C1491 two small dips located at 3.43 and $3.52 \mu \mathrm{m}$ can be observed in Fig. 2. These are instrumental artifacts. Also shown in the figure is a more distinct absorption dip at $4.26 \mu \mathrm{m}$ for sample $\mathrm{C} 1504$ which is due to carbon dioxide in the optical path. The QW with an Sb mole fraction of 0.12 is thus suitable for use as the basis of a light source for a $\mathrm{CO}_{2}$ gas detector based on optical absorption.

Temperature dependent PL measurements were performed on these samples. Figure 3 shows the spectra of $\mathrm{C} 1504$ with temperature ranging from 6 to $210 \mathrm{~K}$. As the temperature increases the main emission peak, which is from the InAsSb/InAs MQW, shows a shift towards lower energy and an intensity decrement. The activation energy of the luminescence quenching is $28.5 \mathrm{meV} .^{9}$ As can be seen in Fig. 2, the InAsSb/InAs MQWs with higher Sb composition have

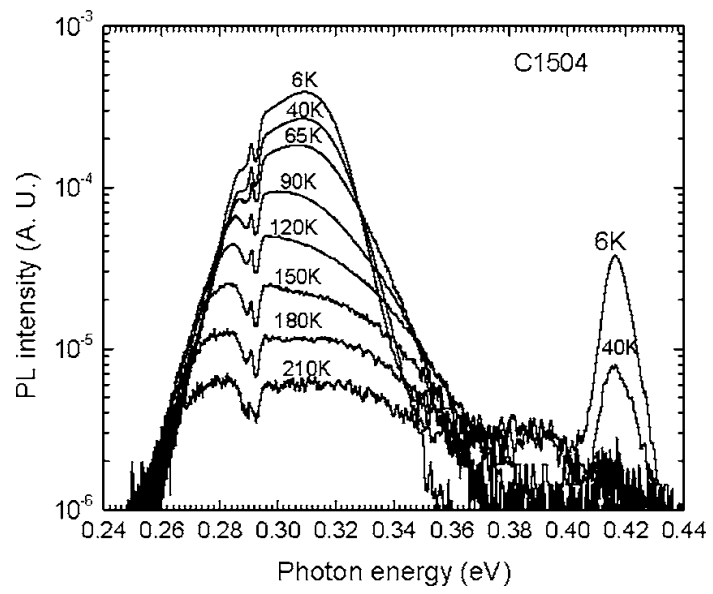

FIG. 3. Photoluminescence spectra of $\mathrm{C} 1504$ at different temperatures ranging from 6 to $210 \mathrm{~K}$.

Downloaded 11 Mar 2008 to 194.80.32.9. Redistribution subject to AlP license or copyright; see http://apl.aip.org/apl/copyright.jsp

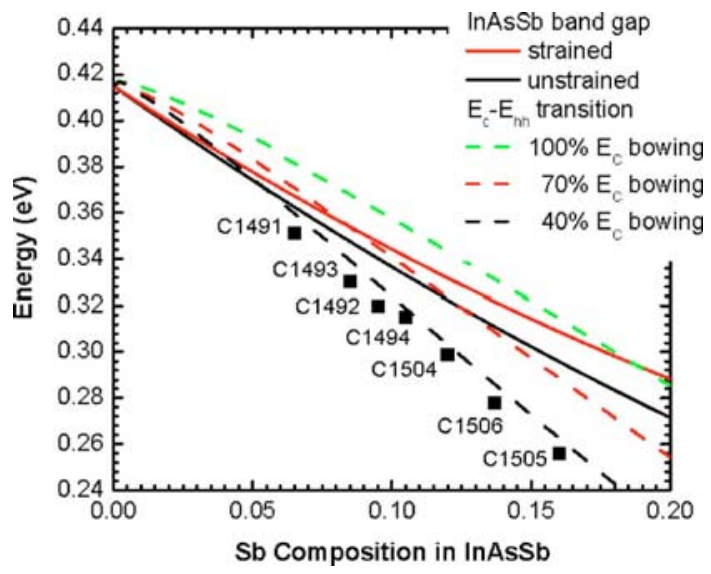

FIG. 4. (Color online) Electron- to heavy-hole transition energy as a function of Sb composition for As-rich InAsSb/InAs MQWs.

a weaker intensity at $4 \mathrm{~K}$. We associate this with a decrease in the radiative recombination coefficient with alloy composition as the $\mathrm{Sb}$ content is increased. However, we observed that increasing the $\mathrm{Sb}$ content in the MQW from $x=0.065$ to 0.12 increases the activation energy for thermal quenching from 14 to $28 \mathrm{meV}$. This is consistent with a progressive reduction in Auger recombination and $\mathrm{CHSH}$ detuning. Besides the main peak, an InAs related peak located at $0.416 \mathrm{eV}$ is also observed. This InAs peak is quenched quickly as the temperature increased. On the low energy side the $\mathrm{CO}_{2}$ absorption was again clearly observed.

Figure 4 shows the peak energies of the InAsSb/InAs MQWs obtained from the $4 \mathrm{~K}$ PL measurements as a function of $\mathrm{Sb}$ composition. The bulk band gap energies of unstrained InAsSb and compressively strained InAsSb are also plotted in the figure. As can be seen, the energies of the MQWs are lower than that of either the unstrained or the strained band gap energy. This clearly implies that the band lineup of the heterostructure is type II. Wei and Zunger ${ }^{8}$ have calculated the band lineup of InAsSb alloys. They indicated that the As-rich InAsSb/InAs is type I when both materials are unstrained and type II when the InAsSb alloy is compressively strained. We further calculated the transition energy from the electron state in InAs to the heavy-hole state in strained InAsSb. Three curves with different band lineups are depicted in Fig. 4. In some reports ${ }^{8,10}$ the band gap bowing in the InAsSb alloy is $100 \%$ attributed to the conduction band. However, in this case the calculated data significantly deviate from the experimental results as can be seen in the figure. This finding implies that the conduction band offset is much larger than that predicted in Ref. 8. As shown in the figure, if we reduce the conduction band bowing to $40 \%$ of the total band gap bowing parameter and associate the remaining $60 \%$ with the valence band, the calculated curve is then in good agreement with the experimental results. We cannot state conclusively that our samples are free of Sb segregation during growth of the InAs layer which could affect the strain as measured using X-ray diffraction. However, Sb segregation would lead to a blueshift in PL which would mean that we have if anything overestimated the conduction band bowing effect.

In summary, we have investigated the photoluminescence of InAsSb/InAs MQW structures. Staggered type-II behavior in the MQWs was observed. By comparing the $4 \mathrm{~K}$
emission peaks of the MQW with a calculation for the
AlP license or copyright; see http://apl.aip.org/apl/copyright.jsp behavior in the MQWs was observed. By comparing the $4 \mathrm{~K}$
emission peaks of the MQW with a calculation for the
AlP license or copyright; see http://apl.aip.org/apl/copyright.jsp 
electron- to heavy-hole transition energy, we found that the band gap bowing could not be totally attributed to the conduction band. For the present case a more accurate situation would be if the bowing parameters of the conduction and valence bands are $40 \%$ and $60 \%$ of the band gap, respectively. For $\mathrm{Sb}$ composition $\sim 0.12$, the emission band covers the absorption energy of $\mathrm{CO}_{2}$ and sources based on InAsSb/InAs MQW could be suitable for use in $\mathrm{CO}_{2}$ gas detector applications.

This work was supported by the Taiwan National Science Council, R.O.C. and the Royal Society under Contract No. NSC-93-2215-E-002-013. The authors also wish to thank D. Campbell and G. Crook for valuable technical assistance.
${ }^{1}$ G. C. Osbourn, J. Vac. Sci. Technol. B 2, 176 (1984).

${ }^{2}$ Z. M. Fang, K. Y. Ma, D. H. Jaw, R. M. Cohen, and G. B. Stringfellow, J. Appl. Phys. 67, 7034 (1990).

${ }^{3}$ Y. H. Zhang, IEEE J. Sel. Top. Quantum Electron. 1, 749 (1995).

${ }^{4}$ B. Lane, D. Wu, A. Rybaltowshi, H. Yi, J. Diaz, and M. Razeghi, Appl. Phys. Lett. 70, 443 (1997).

${ }^{5}$ A. A. Allerman, R. M. Biefeld, and S. R. Kurtz, Appl. Phys. Lett. 69, 465 (1996).

${ }^{6}$ N. V. Zotova, S. S. Kizhaev, S. S. Molchanov, T. B. Popova, and Yu. P. Yakovlev, Semiconductors 34, 1402 (2000).

${ }^{7}$ J. W. Matthews and A. E. Blakeslee, J. Cryst. Growth 27, 118 (1974).

${ }^{8}$ S. H. Wei and A. Zunger, Phys. Rev. B 52, 12039 (1995).

${ }^{9}$ J. I. Pankove, Optical Processes in Semiconductors (Dover, New York, 1971), p. 165

${ }^{10}$ S. Tiwari and D. J. Frank, Appl. Phys. Lett. 60, 630 (1992). 